OPEN ACCESS

Edited by:

Hai-Lei Wei,

Institute of Agricultural Resources and Regional Planning (CAAS), China

Reviewed by:

Wu Xiong,

Utrecht University, Netherlands

Aymé Spor,

INRA UMR 1347 Agroécologie,

France

*Correspondence:

Lioba Rüger

lioba.rueger@uni-koeln.de

Specialty section:

This article was submitted to Microbe and Virus Interactions with Plants,

a section of the journal

Frontiers in Microbiology

Received: 06 October 2020

Accepted: 25 January 2021

Published: 12 February 2021

Citation:

Rüger L, Feng K, Dumack K, Freudenthal J, Chen $Y$, Sun $R$, Wilson $M$, Yu P, Sun B, Deng Y, Hochholdinger $F$, Vetterlein $D$ and

Bonkowski M (2021) Assembly

Patterns of the Rhizosphere Microbiome Along the Longitudinal Root Axis of Maize (Zea mays L.).

Front. Microbiol. 12:614501. doi: 10.3389/fmicb.2021.614501

\section{Assembly Patterns of the Rhizosphere Microbiome Along the Longitudinal Root Axis of Maize (Zea mays L.)}

\author{
Lioba Rüger ${ }^{1 *}$, Kai Feng ${ }^{2,3}$, Kenneth Dumack ${ }^{1}$, Jule Freudenthal', Yan Chen ${ }^{4}$, \\ Ruibo Sun ${ }^{5}$, Monica Wilson', Peng Yu' ${ }^{6}$, Bo Sun ${ }^{4}$, Ye Deng ${ }^{2,3}$, Frank Hochholdinger 6 , \\ Doris Vetterlein ${ }^{7,8}$ and Michael Bonkowski ${ }^{1}$
}

\begin{abstract}
${ }^{1}$ Terrestrial Ecology, Institute of Zoology, University of Cologne, Cologne, Germany, ${ }^{2}$ CAS Key Laboratory for Environmental Biotechnology, Research Center for Eco-Environmental Sciences, Chinese Academy of Sciences, Beijing, China, ${ }^{3}$ College of Resources and Environment, University of Chinese Academy of Sciences, Beijing, China, ${ }^{4}$ Institute of Soil Science, Chinese Academy of Sciences, Nanjing, China, ${ }^{5}$ Microbial Ecology Lab, Center for Agricultural Resources Research, Institute of Genetics and Developmental Biology, Chinese Academy of Sciences, Shijiazhuang, China, ${ }^{6}$ Crop Functional Genomics, Institute of Crop Science and Resource Conservation (INRES), University of Bonn, Bonn, Germany, ${ }^{7}$ Department of Soil System Science, Helmholtz Centre for Environmental Research - UFZ, Halle, Germany, ${ }^{8}$ Soil Science,

Martin-Luther-University Halle-Wittenberg, Halle, Germany
\end{abstract}

It is by now well proven that different plant species within their specific root systems select for distinct subsets of microbiota from bulk soil - their individual rhizosphere microbiomes. In maize, root growth advances several centimeters each day, with the locations, quality and quantity of rhizodeposition changing. We investigated the assembly of communities of prokaryotes (archaea and bacteria) and their protistan predators (Cercozoa, Rhizaria) along the longitudinal root axis of maize (Zea mays L.). We grew maize plants in an agricultural loamy soil and sampled rhizosphere soil at distinct locations along maize roots. We applied high-throughput sequencing, followed by diversity and network analyses in order to track changes in relative abundances, diversity and co-occurrence of rhizosphere microbiota along the root axis. Apart from a reduction of operational taxonomic unit (OTU) richness and a strong shift in community composition between bulk soil and root tips, patterns of microbial community assembly along maize-roots were more complex than expected. High variation in beta diversity at root tips and the root hair zone indicated substantial randomness of community assembly. Root hair zone communities were characterized by massive co-occurrence of microbial taxa, likely fueled by abundant resource supply from rhizodeposition. Further up the root where lateral roots emerged processes of community assembly appeared to be more deterministic (e.g., through competition and predation). This shift toward significance of deterministic processes was revealed by low variability of beta diversity, changes in network topology, and the appearance of regular phylogenetic co-occurrence patterns in bipartite networks between prokaryotes and their potential protistan predators. Such patterns were strongest in regions with 
fully developed laterals, suggesting that a consistent rhizosphere microbiome finally assembled. For the targeted improvement of microbiome function, such knowledge on the processes of microbiome assembly on roots and its temporal and spatial variability is crucially important.

Keywords: archaea, bacteria, Cercozoa, microbial assembly, plant microbiome, protists, rhizosphere

\section{INTRODUCTION}

The predictable assembly of specific subsets of the soil microbiota in the rhizosphere of plants has led to the characterization of plant species-specific "microbiomes" (Hirsch and Mauchline, 2012; Lundberg et al., 2012; Peiffer et al., 2013; Berg et al., 2014). As the microbiome concept implies a rather static outcome of microbial assembly processes, it raises the question as to where and how the dynamic transition of a microbial bulk soil community into a plant species-specific rhizosphere microbiome is taking place.

Differences in resource supply (bottom-up processes) are thought to be the main driver of microbiome assembly (Edwards et al., 2015; van der Heijden and Schlaeppi, 2015). The vast majority of microorganisms in bulk soil rest in an inactive dormant state of starvation, because their activity is severely limited by the availability of energy from readily accessible carbon molecules (Blagodatskaya and Kuzyakov, 2013). This carbon limitation is temporarily offset by pulses of exudates released by the growing root that triggers the bulk soil bacteria into activity (Boddy et al., 2007). Several studies have demonstrated that it takes between 6 and $10 \mathrm{~h}$ until bacteria have switched their metabolism from dormancy to active growth (Anderson and Domsch, 1985; Blagodatskaya et al., 2009; Bonkowski and Clarholm, 2012). However, root exudation does not stimulate rhizobacteria uniformly, rather, rhizodeposition selects for certain fast-growing, copiotrophic bacterial taxa (Maloney et al., 1997; Zelenev et al., 2005; Fierer et al., 2007), leading to reduced taxonomic diversity in the rhizosphere compared to bulk soil (Bulgarelli et al., 2012; Shi et al., 2015; Fan et al., 2017). It is thought that differences in the amount and composition of rhizodeposits further select for the adapted plant speciesand genotype-specific bacterial microbiomes (Bais et al., 2006; Hartmann et al., 2009; Bulgarelli et al., 2012).

The dynamic nature of root systems contrasts with the rather static perception of microbiomes. Plant roots, and the carbon sources they provide, are far from uniform. Rhizodeposition is locally restricted to specific root regions, which undergo continuous transformation (Watt et al., 2006). At their root tips, plants slough off root cap cells (Hawes, 1991; Iijima et al., 2000) and secrete mucilage (Osborn et al., 1999; Knee et al., 2001). Further up the root, small molecular weight exudates are passively released mainly via root hair cells and leak out where laterals emerge (Jaeger et al., 1999; Farrar et al., 2003; Walker et al., 2003; Shaw et al., 2006). Individual primary and seminal roots of maize typically grow around $2-3 \mathrm{~cm}_{\text {day }}{ }^{-1}$ but up to $7 \mathrm{~cm} \mathrm{day}^{-1}$ (Watt et al., 2006; de Moraes et al., 2019) therefore suggesting a continuous process of community reassembly and sustained microbial invasions from bulk soil along the advancing root.
Community assembly theory assumes randomness through priority effects, in which earlier arriving species gain a competitive advantage over subsequent niche inhabitants through exploitation (niche preemption) or manipulation of rhizodeposition (niche modification) along the growing root axis (Fukami, 2015). Randomness through niche preemption can be significantly reduced if competitiveness between taxa differs (Tovi et al., 2019). For example, Lugtenberg et al. (2001) identified the rapid colonization of root tips as a key trait of successful "rhizosphere competent" bacteria, suggesting that microbiome assembly will be shaped already at the root apex. In correspondence Dupuy and Silk (2016), concluded from their model that attachment to root tips was a key bacterial trait that gave bacteria greatest access to exudate carbon and significantly increased their proliferation along the root. In contrast, priority effects through niche modification as shown for the gut microbiome (Sprockett et al., 2018), appear also common in the rhizosphere, where microorganisms influence the composition of root exudates (Naher et al., 2008), change root immune responses and trigger the release of quorum sensing mimics by plant roots (Mathesius et al., 2003; Bauer and Mathesius, 2004; Barnard et al., 2007), or even manipulate the whole root architecture (Hartmann and Schikora, 2012). The early colonizers may modify the environment for laterarriving species to such an extent that assembly history may become the dominant driver of community assembly (Fukami, 2015).

As root growth progresses, the initially random community assembly at the root tip is expected to be replaced at some point by increasingly deterministic processes that lead to the typical plant-associated microbiomes. Deterministic assembly of microbial communities can arise through two mechanisms. The first is competition for resources, a mechanism that is likely to increase when rhizodeposits subside (Nemergut et al., 2013). The second is through selection, by both the plant immune system at the rhizoplane (van der Heijden and Schlaeppi, 2015) and its surrounding rhizosphere where predation of bacterivores (top-down processes) leads to deterministic patterns in reproduction and mortality rates of individual bacterial taxa (Matz and Kjelleberg, 2005; Jousset, 2012). Especially the omnipresent predation of bacterivore protists exerts substantial selection pressure on rhizosphere bacteria (Jousset et al., 2008, 2010; Rosenberg et al., 2009; Henkes et al., 2018), thereby altering functional traits such as increasing grazing resistance and biocontrol compounds (Jousset et al., 2006, 2010; Jousset and Bonkowski, 2010; Jousset, 2012; Flues et al., 2017). However, bacterial investment in defenses carries a significant growth-defense tradeoff (Riley and Wertz, 2002; Denison et al., 2003; Corno 
and Jürgens, 2008; Jousset et al., 2009) thus redirecting competitive outcomes in bacterial communities under selection pressure of protistan bacterivores (Matz and Jurgens, 2003; Jousset et al., 2008, 2009).

To gain insights into the self-organization of the rhizosphere microbiome, we investigated the assembly of rhizosphere specific microbial communities of prokaryotes and their protistan predators along the longitudinal root axis of maize plants at clearly defined root regions. We investigated the early assembly at root tips to communities of subsequently older root regions until first order lateral roots dominated root architecture. We hypothesized an immediate reduction of alpha diversity of prokaryotes in rhizosphere as compared to bulk soil due to a competitive advantage of the fast-growing, copiotrophic taxa from rhizodeposition. Differences in beta diversity, however, should be maximized on root tips, due to an increased likelihood of random priority effects in the early stages of community assembly. Further on, we expected that root regions with highest resource availability (e.g., root tips and the root hair region) would favor many taxa indiscriminately, becoming visible by increased evenness and in networks by high positive co-occurrence of taxa. Finally, we expected the strongest selective forces when rhizodeposits subside, both by microbial competition and predation, which eventually leads to the assembly of the microbiome. Since the "microbiome" is predicated on deterministic processes, it would be characterized by the strongest shift in community structure (beta diversity) compared to bulk soil, together with a strongly reduced variation of beta diversity (i.e., high determinism). At the same time, cooccurrence networks would show a more structured topology, on the one hand due to competitive exclusion and on the other hand by taxa which coevolved during the assembly process and can mutually coexist in the rhizosphere. The existence of topdown processes by protists through selective grazing should be revealed in potential trophic networks between prokaryotes and protists by clear phylogenetic patterns of both negative co-occurrences (i.e., less defended taxa) as well as positive cooccurrences (i.e., well defended taxa benefiting from protists feeding on their competitors).

\section{MATERIALS AND METHODS}

\section{Experimental Set Up}

Zea mays seeds (inbred line B73) were surface sterilized with $10 \% \mathrm{H}_{2} \mathrm{O}_{2}$ for $10 \mathrm{~min}$ and germinated on wet filter paper under sterile conditions at $18^{\circ} \mathrm{C}$ in the dark. After 3 days, 40 seedlings of similar length were selected and separately planted into cylindrical perspex microcosms (250 mm height, $70 \mathrm{~mm}$ inner diameter) filled with $885 \mathrm{~cm}^{3}$ of $1 \mathrm{~mm}$ sieved sandy loam soil at a bulk density of $1.46 \pm 0.1 \mathrm{~g}$ dry wt $\mathrm{cm}^{-3}$, corresponding to $1300 \pm 80 \mathrm{~g}$ dry wt microcosm ${ }^{-1}$. The bottom of each microcosm was closed with a nylon gauze (30 $\mu \mathrm{m}$ mesh size) for watering. Soil water content was kept constant at $22 \%$ vwC by daily weighing and replacement of water. Tubes were wrapped in aluminum foil to protect soil and roots from light. Plants were grown in a climate chamber with a day-night regime of $12 / 12 \mathrm{~h}(350 \mathrm{M}$ PAR $)$ at $24^{\circ} \mathrm{C} / 18^{\circ} \mathrm{C}$ and $65 \%$ humidity.

\section{Sampling}

Microcosms were sampled 9 days after planting. Using sterile forceps and scissors, $1 \mathrm{~cm}$ root samples with adhering soil from (i) the root tip, (ii) the root hair zones, (iii) the region where the first lateral root primordia emerged as earliest lateral roots, and (iv) from a subsequent region with fully developed lateral roots (Figure 1) were transferred into sterile $15 \mathrm{ml}$ centrifuge tubes. Samples of three different roots of the same plant were pooled to one biological replicate. As a control, a bulk soil sample was taken from each microcosm, consisting of five randomly chosen and pooled soil samples which were not in the direct vicinity of a root. Each root region and bulk soil sampling was replicated 40 times. Soil was washed off from roots by vortexing in a $0.3 \% \mathrm{NaCl}$ solution. After $30 \mathrm{~min}$ of centrifugation at $5000 \times \mathrm{g}$, the supernatant was discarded, and the pellet was used for DNA extraction. DNA-extraction and purification were done using the FastDNA $^{\circledR}$ SPIN Kit for soil and the GENECLEAN ${ }^{\circledR}$ SPIN Kit (MP Biomedicals, Santa Ana, CA, United States), following the manufacturer's instructions.

\section{Cercozoa}

\section{Amplicon-Sequencing}

A two-step PCR yielding a c. 350 bp long fragment of the $\mathrm{V} 4$ region of the SSU/18S gene was conducted using primers (Fiore-Donno et al., 2020) targeting amplification of Cercozoa. In a first PCR a mixture of identical amounts of the forward primers S615F_Cerco (5'-GTTAAAAAGCTCGTAGTTG-3') and S615F_Phyt (5'-GTTAAAARGCTCGTAGTCG-3') together with the reverse primer S963R_Phyt (5'-CAACTTTCGT TCTTGATTAAA- $3^{\prime}$ ) were used. In a second, semi-nested PCR, samples were indexed by using the forward primer S615F_Cer (5'GTTAAAARGCTCGTAGTYG-3') and the reverse primer S947R_Cer (5'-AAGARGAYATCCTTGGTG-3'), both tagged with barcodes as described by Fiore-Donno et al. (2020).

A $1 \mu l$ of purified DNA from soil samples served as the template for the first PCR, resulting in amplicons of which $1 \mu \mathrm{l}$ was used for the second semi-nested PCR. Both PCR-rounds were conducted with reagents in the following final concentrations: Dream Taq polymerase (Thermo Fisher Scientific, Dreieich, Germany) 0.01 units, Thermo Scientific Dream Taq Green Buffer, dNTPs $0.2 \mathrm{mM}$ and primers $1 \mu \mathrm{M}$. All PCRs were conducted in duplicates to reduce the possible artificial dominance of few amplicons by PCR competition and then pooled. The thermal program for the reaction started with a 2 min denaturation step at $95^{\circ} \mathrm{C}$, followed by $95^{\circ} \mathrm{C}$ for $2 \mathrm{~min},\left(95^{\circ} \mathrm{C}\right.$ for $30 \mathrm{~s}, 50^{\circ} \mathrm{C}$ for $30 \mathrm{~s}, 72^{\circ} \mathrm{C}$ for $30 \mathrm{~s}$ ) repeated 24 times and a final elongation step at $72^{\circ} \mathrm{C}$ for $5 \mathrm{~min}$. After checking for successful amplification and potential contamination by gel electrophoresis, $24 \mu \mathrm{l}$ of the PCR-product of each sample were purified and normalized using SequalPrep Normalization Plate Kit (Invitrogen $\mathrm{GmbH}$, Karlsruhe, Germany) to obtain a concentration of $1-2 \mathrm{ng} / \mu \mathrm{l}$ per sample. Purified amplicons were pooled, concentrated and sequenced on an Illumina MiSeq platform (Illumina Inc., San Diego, CA, United States) at the Cologne Center for 


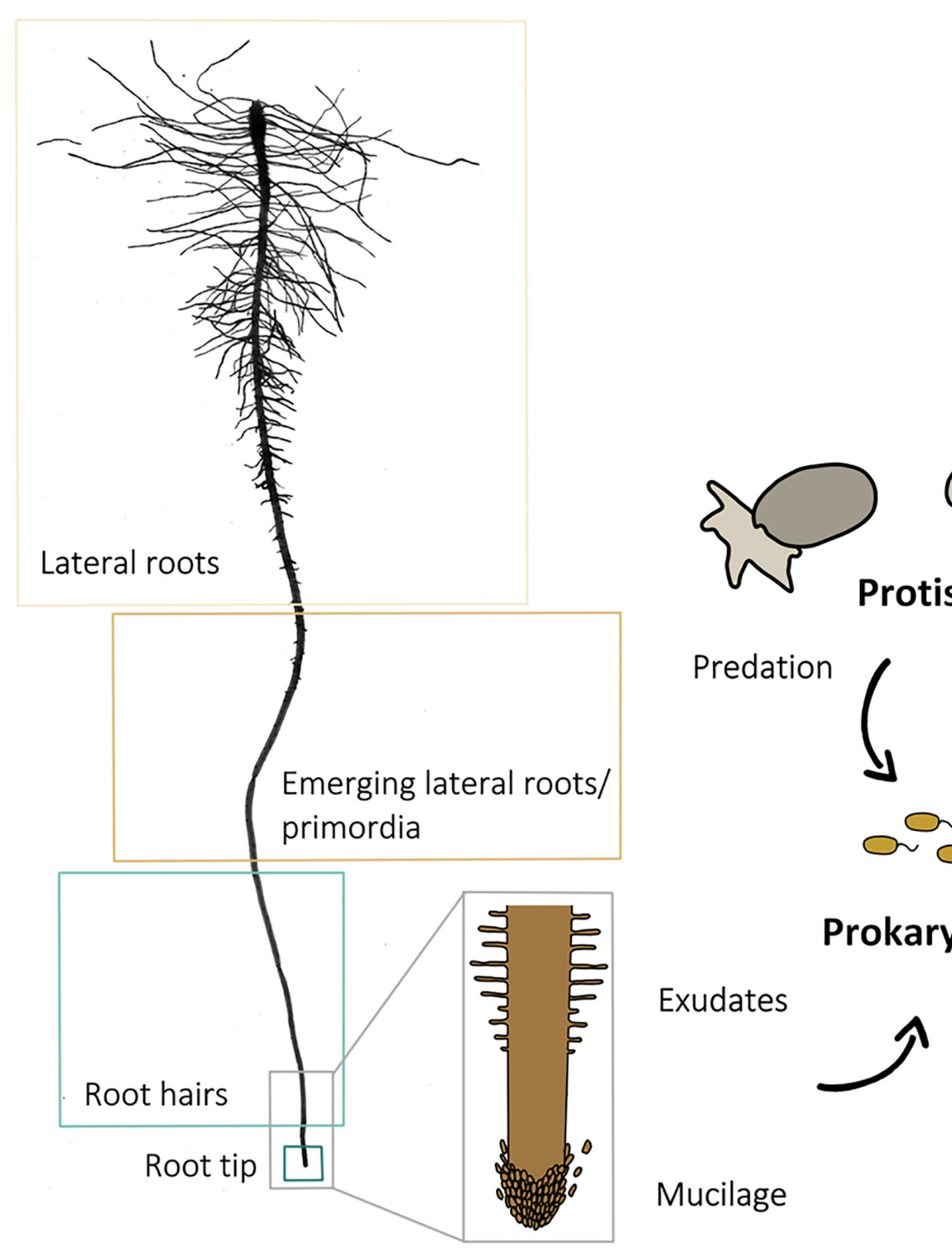

FIGURE 1 | Scan of a washed primary root of an experimental plant and scheme of a root with root cap cells at the tip and root hairs above. The arrows indicate the direction of the influence different components (root, prokaryotes, and protists) exert. The four sampled root regions (lateral roots, lateral root primordia, root hair zone, and root tip) are framed for identification.

Genomics (Cologne, Germany). With the MiSeq v3 Reagent kit, $2 \times 300$ cycles were performed, producing 300 bp long paired-end reads.

\section{Sequence Processing}

Reads were processed using the customized MOTHUR pipeline v.39.5 (Schloss et al., 2009).

Paired-end reads were merged, not allowing any mismatches in primer or barcode sequences, maximum two mismatches and one ambiguity in the target sequence. Assembled sequences with an overlap $<200$ bp were removed. Merged contigs were demultiplexed and primer and tag sequences were trimmed. Remaining reads were clustered into operational taxonomic units
(OTUs) using VSEARCH (Rognes et al., 2016) according to the abundance-based greedy algorithm (agc) with a similarity threshold of $97 \%$. Clusters represented by less than 500 reads were removed as likely to represent amplification or sequencing noise (Fiore-Donno et al., 2018). OTUs were assigned to taxa using BLAST+ (Camacho et al., 2009) with an e-value of $1^{e-50}$ and the PR2 database (Guillou et al., 2013), keeping only the best hit. Non-target sequences (12 of 723 OTUs were excluded. Sequences were aligned with the provided template (FioreDonno et al., 2018), allowing gaps of maximum five nucleotides and cleaned from chimeras using UCHIME (Edgar et al., 2011), resulting in 513 OTUs. The sampling depth reached up 17,062 sequences per sample. 


\section{Bacteria/Archaea \\ Amplicon-Sequencing}

The forward primer 515F (5'-GTGCCAGCMGCCGCGGTAA-

$3^{\prime}$ ) (Caporaso et al., 2011) and the reverse primer 806R (5'-GGACTACNVGGGTWTCTAAT-3') (Apprill et al., 2015), targeting a c. $390 \mathrm{bp}$ long fragment of the V4 region of the SSU/16S genes of bacteria and archaea were used for amplification. The PCR was set up with $2 \mu \mathrm{l}$ of template DNA, $12.5 \mu \mathrm{l}$ of TaKaRa Premix Taq ${ }^{\mathrm{TM}}$ (TaKaRa Bio Group) and 10 $\mu \mathrm{M}$ of forward and reverse primer each. The thermal program for the reaction started with a $5 \mathrm{~min}$ denaturation step at $95^{\circ} \mathrm{C}$, followed by $\left(94^{\circ} \mathrm{C}\right.$ for $30 \mathrm{~s}, 56^{\circ} \mathrm{C}$ for $30 \mathrm{~s}, 72^{\circ} \mathrm{C}$ for $\left.40 \mathrm{~s}\right)$ repeated 30 times and a final elongation step at $72^{\circ} \mathrm{C}$ for $5 \mathrm{~min}$. The sequencing was done on the Illumina HiSeq platform (Illumina Inc., San Diego, CA, United States) by Magigene Technology Co., Ltd. (Guangzhou, China). With a HiSeq v2 Reagent kit, $2 \times 250$ cycles were performed, producing $250 \mathrm{bp}$ long paired-end reads.

\section{Sequence Processing}

Quality trimming and adapter clipping of the raw reads was performed using Trimmomatic (Bolger et al., 2014). Pairedend reads were merged using fastq-join (Aronesty, 2011) with a minimum overlap of $10 \mathrm{bp}$ and maximum $10 \%$ difference within the overlapping region. Reads containing errors in primer sequences were discarded, and primer sequences were removed using cutadapt (Martin, 2011). Chimeric reads were filtered and removed with UCHIME (Edgar et al., 2011). Subsequently VSEARCH (Rognes et al., 2016) was used to cluster reads into OTUs with a similarity threshold of $97 \%$. To assign taxa to OTUs the RDP Classifier was used with the Silva database (version 132) as reference. OTUs which were not assigned to bacteria or archaea as well as those represented by less than 100 reads were removed to get rid of erroneous sequences. Read counts were subsampled to the minimum number of reads $(50,438)$ in a sample.

\section{Statistical Analysis}

Statistical analyses and data visualization were performed in $\mathrm{R}$ version 3.5.1 ( $\mathrm{R}$ Core Team, 2018). With our sequencing depth we reached saturation in taxon sampling (Supplementary Figure 1). For downstream analysis, the total number of reads was transformed into relative abundances. Species richness, evenness and alpha diversity were compared using Welch's oneway ANOVA, followed by a Games-Howell non-parametric post hoc test. After the removal of one outlier from the prokaryote and two from the cercozoan dataset permutational multivariate analysis of variance (PERMANOVA) using BrayCurtis dissimilarity, permuted 999 times, was employed to test for differences in prokaryote and cercozoan community structure across samples from different root regions.

\section{Network Analysis}

Co-occurrence networks incorporating communities containing bacteria, archaea, and Cercozoa were based on single OTUs and generated to assess co-occurrence or potential interactions between species. To assess the complexity and specificity of community structures along roots, network analyses were conducted for communities at the four different sampled root regions and from bulk soil. For network construction and analysis of topological features the molecular ecological network analysis pipeline (MENAP) ${ }^{1}$ (Zhou et al., 2011; Deng et al., 2012) was used. From OTUs which occurred in more than $75 \%$ of the samples within each group a Spearman rank correlation matrix without log-transformation was calculated. Based on random matrix theory a threshold of 0.76 was determined in MENAP. When the correlation coefficients were higher than 0.76 , interactions were considered as significant positive, when they were lower than -0.76 as significant negative. As implemented in the MENAP topological features as total number of nodes (OTUs), total number of links, $R^{2}$ of power law, average connectivity (or average degree) which measures the complexity of a network, average clustering coefficient, average path distance and modularity were calculated. Based on detected modules among-module connectivity and inter-module connectivity were calculated, and nodes were assigned to one of four possible network roles: network hubs, module hubs, connectors or periphers.

In order to characterize the potential impact of protists on prokaryote community assembly along roots, inter-domain associations between Cercozoa and prokaryotes were extracted from full networks. From these, bipartite networks were generated with nodes displaying cercozoan OTUs, grouped at family and prokaryote OTUs, grouped at phylum level. The network topological features of these bipartite networks were calculated using the Interdomain Ecological Network Analysis Pipeline (IDENAP) ${ }^{2}$, including connectance which is the proportion of possible links that are established, links per OTU, number of compartments, and modularity for the cercozoan community. The network graphs were visualized in Cytoscape 3.7.2 (Shannon et al., 2003).

\section{RESULTS}

\section{Community Structure}

After sequence processing 513 cercozoan and 3355 prokaryotic OTUs were obtained. OTU richness of prokaryotes was highest in bulk soil, but dropped significantly when encountered by a root tip, and then only gradually increased again toward the older root regions (Figure 2, ANOVA, $F_{4}, 186=14.12$, $p<0.001$ ). Bulk soil and root tips showed highest prokaryote alpha diversity (ANOVA, $F_{4}, 186=12.88, p<0.001$ ) and evenness (ANOVA, $F_{4}, 186=16.71, p<0.001$ ) compared to older root regions (Figure 2). However, OTU richness, alpha diversity and evenness of root-associated prokaryotes showed substantially higher variation than in bulk soil (Supplementary Table 1). In contrast to prokaryotes, protist richness and alpha diversity did not differ between bulk soil and root regions (Figure 2).

Beta diversity differed significantly between bulk soil and root regions and among different root regions, in both prokaryotes $\left(\right.$ PERMANOVA $\left., F_{4}, 185=12.13, R^{2}=0.22, p<0.001\right)$

\footnotetext{
${ }^{1}$ http://ieg4.rccc.ou.edu/mena/

${ }^{2}$ http://mem.rcees.ac.cn:8081
} 


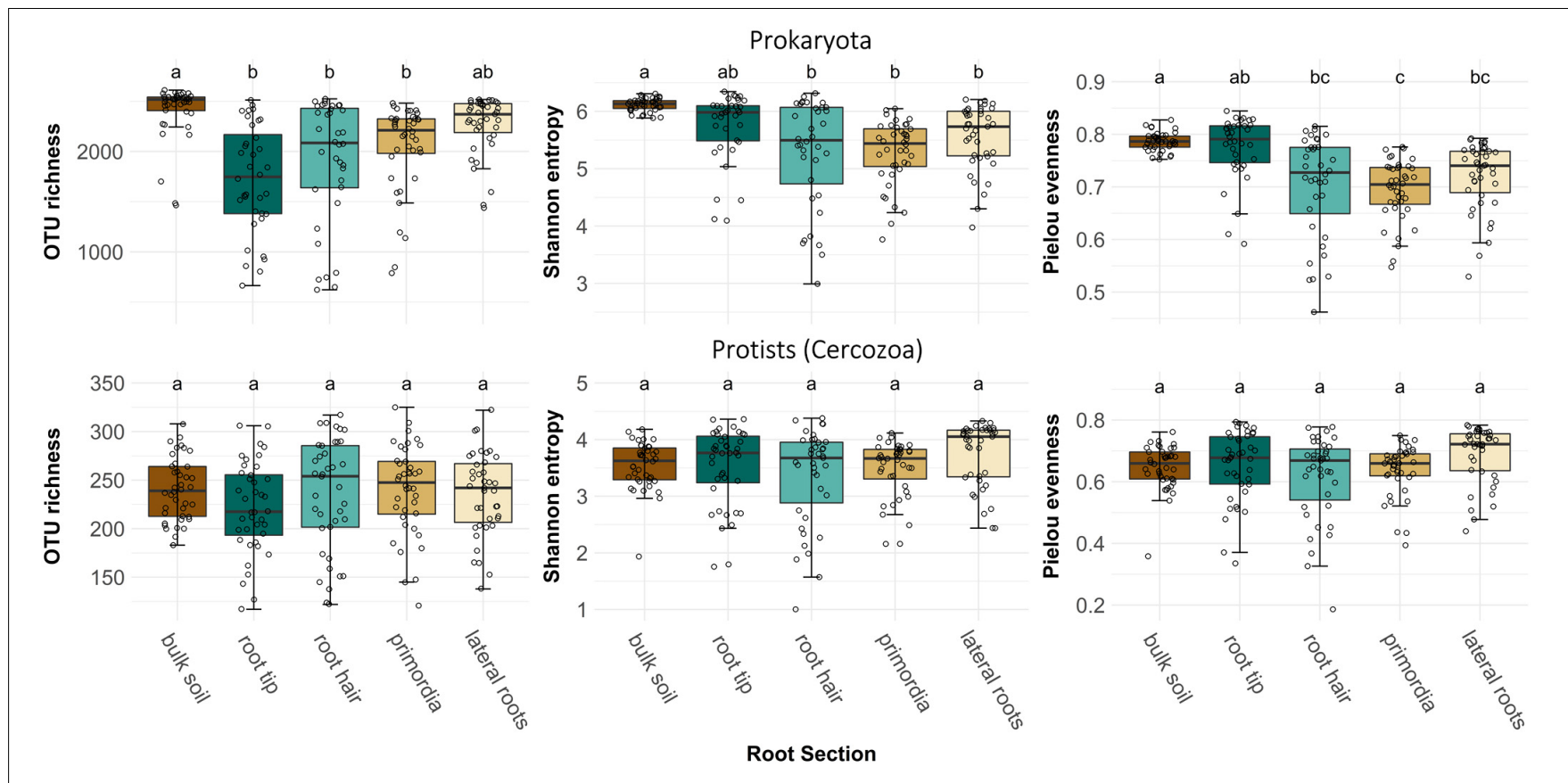

FIGURE 2 | Operational taxonomic unit richness, alpha diversity (Shannon index) and evenness (Pielou's index) of prokaryote and protist (cercozoan) communities in bulk soil and at four different root regions of maize plants. Different letters indicate differences between means (Welch's one-way with Games-Howell post hoc test). Prokaryote OTU richness: $F_{4,186}=14.12, p<0.001$, prokaryote Shannon diversity: $F_{4,186}=12.88, p<0.001$, prokaryote Pielou's evenness: $F_{4,186}=16.71$, $p<0.001$, protist OTU richness: $F_{4,193}=1.09, p>0.05$, protist Shannon diversity: $F_{4,193}=1.99, p>0.05$, and protist Pielou's evenness: $F_{4,193}=2.62, p=0.36$.

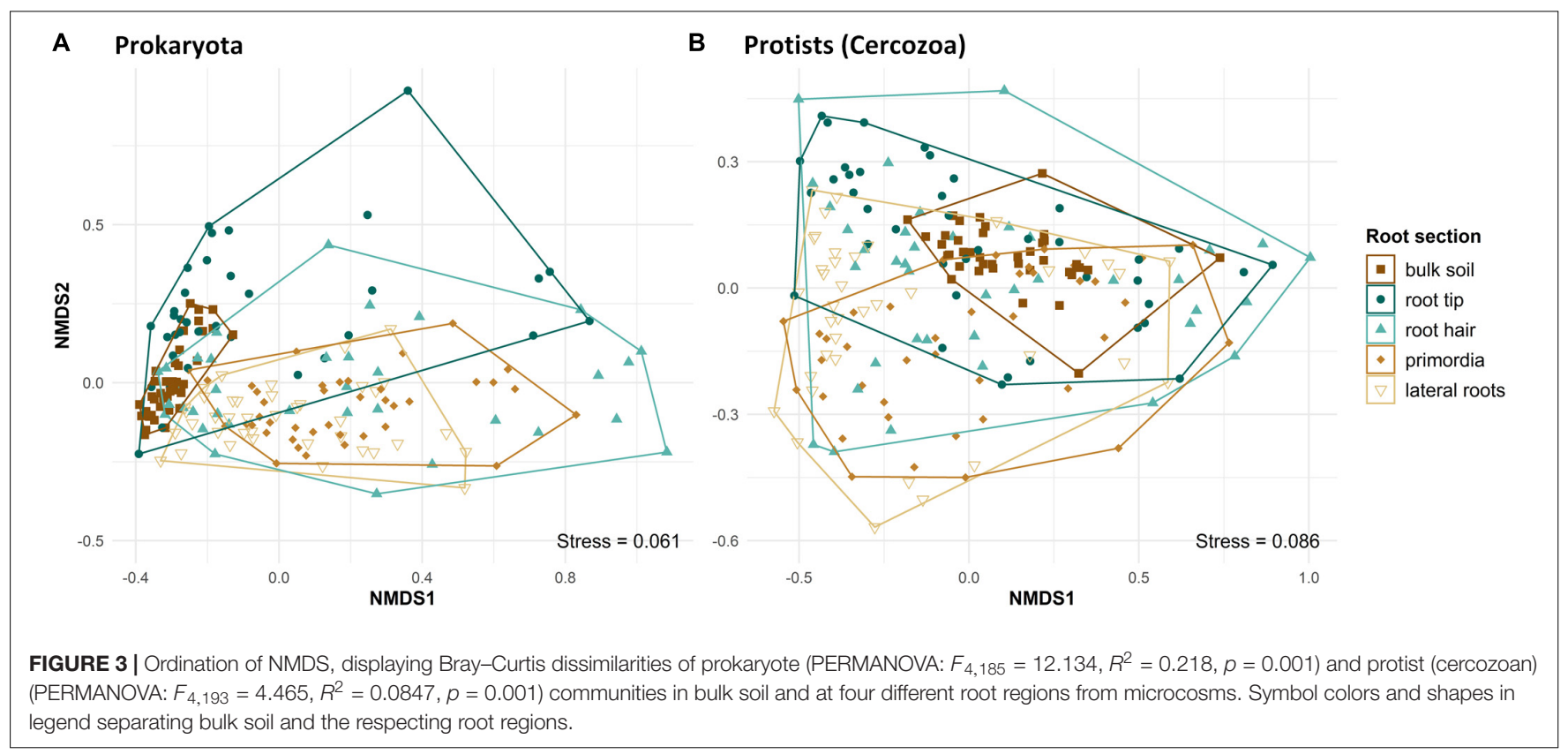

and protists (PERMANOVA, $F_{4}, 193=4.47, R^{2}=0.085$, $p<0.001)$. Beta diversity of prokaryote communities was lowest in bulk soil, highest at root tips and the root hair zone, and decreased again toward lateral root primordia and lateral roots, respectively (Figure 3, NMDS plot). Pairwise comparisons revealed differences in community structure of prokaryotes in bulk soil and at root tips compared to all other root regions and between the communities of lateral root primordia compared to lateral roots (Supplementary Table 2). Protist community structure differed significantly between bulk soil and root tips (Supplementary Table 3) and generally reflected the pattern seen in NMDS of prokaryotes although shifts in beta diversity from root tips to increasingly older root regions showed more variation (Figure 3B). 
TABLE 1 | Topological features of empirical networks of bulk soil, root tip, root hair zone, lateral root primordia and lateral roots, including inter inter- and intra-domain and intra domain co-occurrences of bacteria, archaea, and Cercozoa.

\begin{tabular}{|c|c|c|c|c|c|c|c|c|}
\hline & \multicolumn{8}{|c|}{ Empirical networks } \\
\hline & $\begin{array}{l}\text { Similarity } \\
\text { threshold }\end{array}$ & $\begin{array}{c}\text { Total number of } \\
\text { nodes }\end{array}$ & $\begin{array}{l}\text { Total number of } \\
\text { links }\end{array}$ & $\begin{array}{c}R^{2} \text { of } \\
\text { power-law }\end{array}$ & $\begin{array}{c}\text { Average degree } \\
\text { (avgK) }\end{array}$ & $\begin{array}{l}\text { Average clustering } \\
\text { coefficient }(\operatorname{avgCC})^{b}\end{array}$ & $\begin{array}{c}\text { Average path } \\
\text { distance (APD) }\end{array}$ & $\begin{array}{l}\text { Modularity } \\
\text { (MOD) }^{\mathrm{b}}\end{array}$ \\
\hline Bulk soil & 0.76 & 172 & 597 & 0.954 & 6.942 & $0.411^{a}$ & $3.246^{a}$ & $0.553^{a}$ \\
\hline Root tip & 0.76 & 211 & 279 & 0.994 & 2.645 & $0.181^{a}$ & $7.146^{a}$ & $0.787^{\mathrm{a}}$ \\
\hline Root hair & 0.76 & 511 & 6396 & 0.970 & 25.033 & $0.473^{a}$ & $2.914^{a}$ & $0.280^{\mathrm{a}}$ \\
\hline Primordia & 0.76 & 218 & 674 & 0.978 & 6.183 & $0.348^{a}$ & $3.643^{a}$ & $0.511^{a}$ \\
\hline Lateral & 0.76 & 296 & 1521 & 0.988 & 10.277 & $0.405^{a}$ & $3.376^{a}$ & $0.431^{a}$ \\
\hline
\end{tabular}

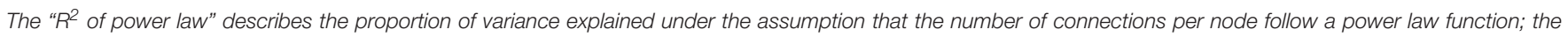
"average degree" is the average number of connections per node in the respective network.

a Significant difference ( $p<0.001)$ in avgCC, APD, and MOD of empirical networks compared to random networks (Table 3), based on one sample Student's $t$ test.

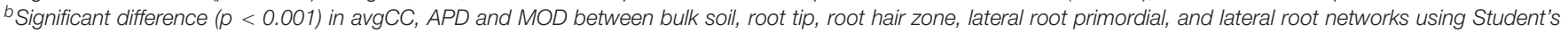
t test.

\section{Network Analysis}

Network topologies of bulk soil and the four root regions differed significantly from randomly generated networks (Tables 1, 2). Network connectivity followed a power law $\left(R^{2}\right.$ from 0.954 to 0.994 ), indicative of a scale-free network structure with a topology of many nodes with few connections and some highly connected nodes within modules (i.e., small values of average path distance, except at root tips; Table 1). The total number of nodes was lowest in bulk soil (172; Table 1) and reached a threefold higher maximum in the root hair zone (511; Table 1). Furthermore, the total number of links was lowest at root tips (279 links; Table 1) but increased 23-fold in the subsequent root hair zone (6396 links, Table 1). Average degree and the average clustering coefficient were lowest and average path distance was highest in root tip networks (Table 1), indicating poorly structured, weakly correlated and widely dispersed networks with few links (Figure 4). In contrast, networks of the root hair zone showed by far the highest average degree, indicating the highest connectivity of OTUs, but the lowest modularity and average path distance (Table 1) suggesting a barely sub-structured and rather dense network with high numbers of co-occurring taxa (Figure 4). Network complexity again substantially decreased at sites of lateral root primordia although by far not as much as at root tips, and finally increased again significantly in the lateral root zone with $16 \%$ lower modularity but $1.38,2.26$, and 1.65 fold increased numbers of nodes, links and average connectivity (avgK) compared to root primordia, respectively (Table 1). Positive co-occurrences by far dominated in all networks (Figure 4), but from bulk soil to lateral root primordia the percentage of negative correlations gradually decreased and only increased again to bulk soil level at sites with fully developed lateral roots. Most nodes were represented by bacteria, while Cercozoa and archaea made up a much smaller share of total nodes (Figures 4A-E). Archaea formed clear sub-network clusters in bulk soil and on lateral roots (Figures 4A,E).

Bacterial taxa exclusively acted as module hubs and network hubs in all five networks (Figure 5). Module hubs were represented by Actinobacteria, Bacteroidetes, and Firmicutes in bulk soil and Proteobacteria, Bacteriodetes, and Acidobacteria in rhizosphere networks. Network hubs were exclusively represented by Acidobacteria in the root hair zone (Figure 5). Cercozoan protists acted as connectors in the more complex community networks of root hair zone and lateral roots, together with a changing variety of bacterial taxa in different root regions (Figure 5).

In order to identify potential trophic relationships between protists and prokaryotes, bipartite inter-domain associations between Cercozoa and prokaryotes were extracted from full networks along roots (Figure 6 and Table 3). The bulk soil bipartite "trophic network" revealed distinct phylogenetic patterns with positive co-occurrences of protists in Allapsidae and Leptophryidae with bacterial members of Bacteriodetes and negative correlations with Proteobacteria and Firmicutes (Figure 6). Although bipartite networks of root tips, the root hair zone and lateral root primordia always identified the same protistan taxa as potential predators, these networks appeared scattered and did not reveal distinct, repeatable patterns of cooccurrence with prokaryotes.

In contrast, the highest complexity of bipartite networks was found on lateral roots, as in bulk soil showing again clearly positive co-occurrences of protists in Allapsidae, Cercomonadidae, and Paracercomonadidae with Bacteriodetes and negative correlations with Proteobacteria (Figure 6E). The percentage of negative correlations in bipartite trophic networks was always quite high, indicating potential predation effects, except in the root hair zone and lateral root region (Figure 6, bar chart). High numbers of prokaryotic OTUs, connectance, and links per species in bulk soil, declined at the root tip, remained low in the root hair zone and sites of lateral root primordia, but significantly increased at lateral roots (Table 3 ) indicating a decrease in network complexity from bulk soil to young root regions, followed by a large increase in network complexity at lateral roots.

\section{DISCUSSION}

The concept of a "core" microbiome implies highly deterministic processes in the assembly of rhizosphere communities and 
TABLE 2 | Means of topological features of 100 networks, generated by randomly rewiring nodes and links of empirical bulk soil, root tip, root hair zone, lateral root primordial, and lateral root networks.

\begin{tabular}{lccc}
\hline \multicolumn{3}{c}{ Random networks } \\
& $\begin{array}{l}\text { Average clustering } \\
\text { coefficient (avgCC) }\end{array}$ & $\begin{array}{c}\text { Average path } \\
\text { distance (APD) }\end{array}$ & $\begin{array}{c}\text { Modularity } \\
\text { (MOD) }\end{array}$ \\
\hline Bulk soil & $0.14 \pm 0.014$ & $2.883 \pm 0.045$ & $0.314 \pm 0.005$ \\
Root tip & $0.017 \pm 0.006$ & $4.455 \pm 0.113$ & $0.657 \pm 0.007$ \\
Root hair & $0.321 \pm 0.008$ & $2.531 \pm 0.013$ & $0.133 \pm 0.003$ \\
Primordia & $0.132 \pm 0.013$ & $3.039 \pm 0.043$ & $0.334 \pm 0.005$ \\
Lateral & $0.202 \pm 0.011$ & $2.78 \pm 0.029$ & $0.234 \pm 0.005$ \\
\hline
\end{tabular}

predictability of their plant-associated traits (Friesen et al., 2011; Oyserman et al., 2018). Recent studies have revealed much about the deterministic nature of species-specific rhizosphere microbiomes and their variation due to differences in soil type, plant developmental stage or plant genotype (Bouffaud et al., 2012; Peiffer et al., 2013; Chaparro et al., 2014; Bulgarelli et al., 2015; Edwards et al., 2015; Perez-Jaramillo et al., 2016, 2017; Rossmann et al., 2020), but much less is known on where and when a rhizosphere microbiome is formed along the root axis from a random bulk soil community (Di Battista-Leboeuf et al., 2003).

It has been assumed that microbiome members are already selected at root tips through mucilage and root border cells (Hawes et al., 2012; Baetz and Martinoia, 2014), while others emphasized the role of root exudates released at more distal regions along the root axis (Oger et al., 2004; Steinkellner et al., 2007; Hartmann et al., 2009; Li et al., 2016), or proposed that the plant microbiome assembles along the way from rhizosphere to endosphere, with the plant immune system at the rhizoplane playing a dominant role for community selection (Lundberg et al., 2012; Edwards et al., 2015).

Community assembly of the maize microbiome along the root axis showed more complex patterns than anticipated. Apart from OTU richness, which strongly decreased at root tips and slowly recovered toward older root regions, there was no gradual transitions of community composition along the longitudinal

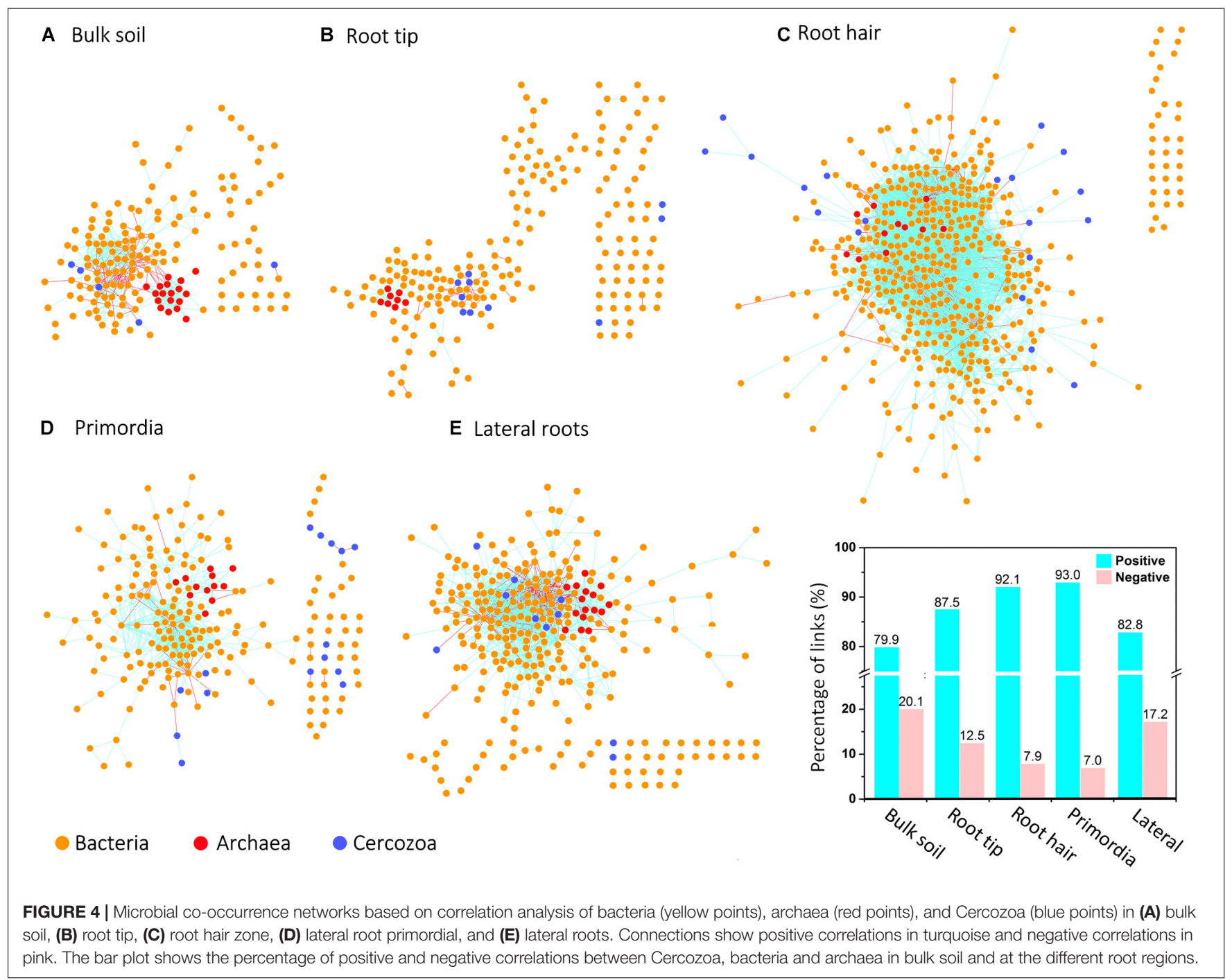




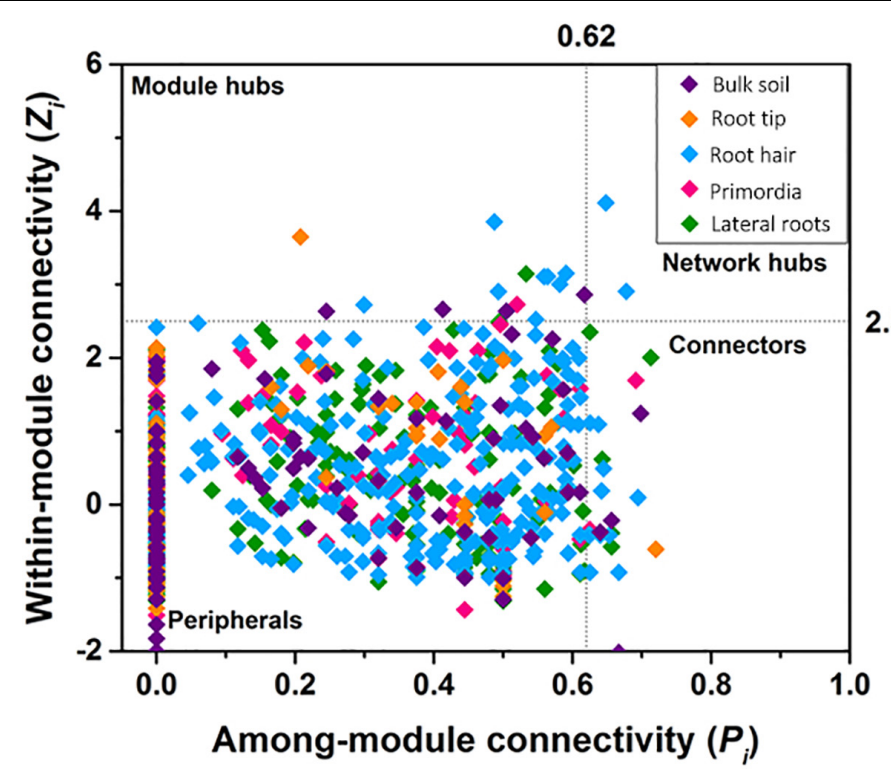

Module hubs: Connectors:

Actinobacteria Actinobacteria*3

Bacteroidetes ${ }^{*}$ Proteobacteria

Firmicutes

Proteobacteria

Acidobacteria*2

Bacteroidetes

2.5 Proteobacteria*5

Proteobacteria

Proteobacteria

Acidobacteria

Acidobacteria

Chloroflexi²

Gemmatimonadetes

Nitrospirae*2

Proteobacteria*6

Verrucomicrobia

Cercozoa

Acidobacteria

Firmicutes

Network hubs: Acidobacteria*2

Acidobacteria*2 Planctomycetes

Proteobacteria

Cercozoa

FIGURE 5 | Within-module connectivity $\left(Z_{\mathrm{i}}\right)$ and among-module connectivity $\left(P_{\mathrm{i}}\right)$ of network nodes. Points exceeding thresholds of $Z_{\mathrm{i}}=2.5$ and/or $P_{\mathrm{i}}=0.62$ were identified as module hubs, network hubs or connectors.

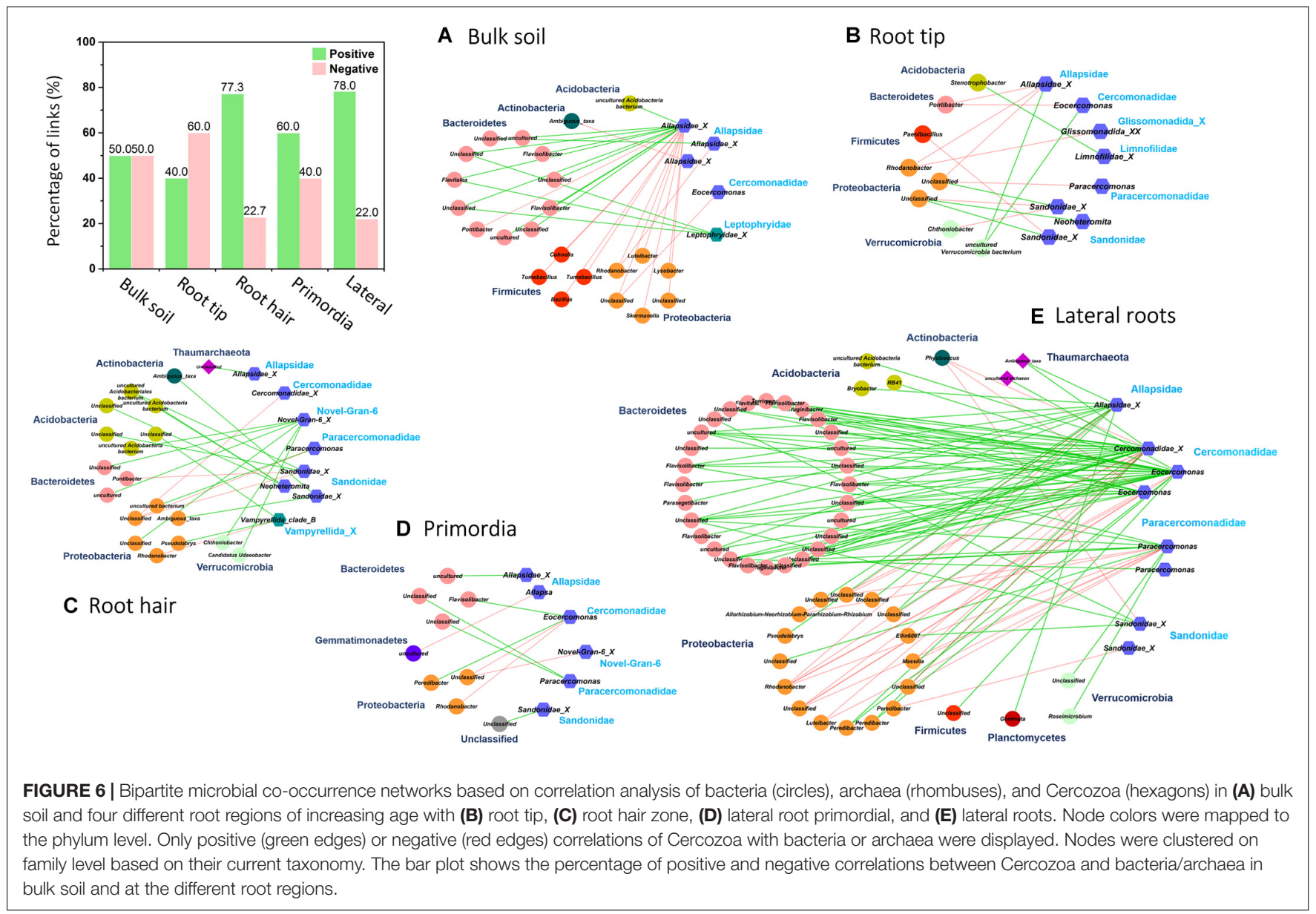


TABLE 3 | Topological features of bipartite networks, only considering co-occurrences of protists with prokaryotes of bulk soil, root tip, root hair zone, lateral root primordial, and lateral roots.

\begin{tabular}{|c|c|c|c|c|c|c|}
\hline & $\begin{array}{c}\text { Number of } \\
\text { protist OTUs }\end{array}$ & $\begin{array}{l}\text { Number of bacterial/ } \\
\text { archaeal OTUs }\end{array}$ & $\begin{array}{c}\text { Total number } \\
\text { of nodes }\end{array}$ & $\begin{array}{l}\text { Total number of } \\
\text { associations }\end{array}$ & Connectance & Links per OTU \\
\hline Bulk soil & 5 & 23 & 28 & 28 & 0.243 & 1.000 \\
\hline Root tip & 8 & 8 & 16 & 15 & 0.234 & 0.938 \\
\hline Root hair & 8 & 19 & 27 & 22 & 0.145 & 0.815 \\
\hline Primordia & 6 & 9 & 15 & 10 & 0.185 & 0.667 \\
\hline Lateral & 8 & 54 & 62 & 109 & 0.252 & 1.758 \\
\hline
\end{tabular}

Connectance is the proportion of possible links that are established.

root axis. In order to understand the mechanisms of microbiome assembly, patterns of microbial alpha and beta diversity, as well as changes in the overall and trophic network structure, had to be equally taken into account.

At root tips, a decrease of OTU richness and alpha diversity of bacteria as compared to bulk soil has been repeatedly reported (Bulgarelli et al., 2012; Shi et al., 2015; Fan et al., 2017). The associated changes in species composition on maize root tips were confirmed by a clear shift in beta diversity compared to bulk soil (Figure 3A), and most likely can be attributed to the dominance of fast-growing copiotrophic users of energy-rich rhizodeposits (Schönwitz and Ziegler, 1989; Andrews and Harris, 2000; Benizri et al., 2007; Fierer et al., 2007). Additionally, there is growing evidence that root border cells and secreted mucilage at tips actively bind, immobilize and aggregate specific microbial cells before they become members of the microbiome (Humphris et al., 2005; Hawes et al., 2012; Baetz and Martinoia, 2014). Root tip communities, however, were characterized by the largest variation in OTU richness and beta diversity (Figures 2, 3B). This pattern suggests that different species gain dominance on individual root tips, demonstrating a substantial randomness in the outcome of early rhizosphere community assembly. These results are further supported by high evenness of prokaryote communities on root tips (Figure 2), indicative of a reduced dominance of specific taxa. Taken together, community structure had markedly changed from bulk soil to root tips, but rhizosphere microbiome composition was far from uniform at the early stages of community assembly.

Maize root exudation contributes 10-100 times more carbon to the rhizosphere than border cells and mucilage at root tips (Nguyen, 2003). Piñeros et al. (2002) reported a maximum release of organic acids by maize exudates about $5 \mathrm{~cm}$ beyond maize root tips. Thus, microorganisms in the root hair zone profit from maximum energy supply of rhizodeposition. Because root exudates contain a variety of plant species-specific metabolites and signal compounds with important functional roles in plant defense and symbiosis (Baetz and Martinoia, 2014) they have been suggested as a main driver for the selection of rootspecific microorganisms (Badri and Vivanco, 2009; Hartmann et al., 2009; van Dam and Bouwmeester, 2016). However, DiniAndreote et al. (2015) hypothesized that microbial dispersal in systems with abundant resource supply will lead to the dominance of neutral, stochastic assembly processes. A marked shift of beta diversity in the root hair zone compared with root tips reflects these rhizosphere-driven changes in community structure (Figure 3A). Nevertheless, maximum variation of OTU richness, alpha diversity, evenness (Figure 2), and high variation of beta diversity (Figure 3A) still show significant randomness in community assembly. An explanation is given by the root hair network structure (Figure 4C). Its low modularity with highest number of nodes and links reflects a large network with massive co-occurrences of taxa (Table 1), indicating a parallel unconstrained growth of large numbers of different microorganisms fueled by the high rhizodeposition (Figure 4C). Accordingly, selective forces through competition and predation will be at a minimum, and this is reflected in the bipartite trophic network of root hairs (Figure 6C). Although the bipartite trophic network indicates the co-occurrences of common protistan taxa with specific bacteria, its network structure was rather poor. It showed the lowest connectance of all root region networks, and the ratio of empirical network links to potential trophic links in the bipartite network increased more than 10-fold compared to bulk soil and root tips, demonstrating that predators were loosely linked to the overall network structure. Protistan beta diversity showed highest variation in the root hair zone (Figure 3B), indicating high variability in the dominance structure of predator communities among replicates. Thus despite the extraordinarily high co-occurrence of prokaryotes in the root hair zone, correlations of prokaryotes to protistan predators were sparse (Figure 6C and Table 3), suggesting that the predators followed an opportunistic feeding strategy and most likely exerted no topdown control over the exponential growth of bacteria. These assumptions are further supported by the high percentage of positive co-occurrences between protists and their potential prey in these networks (Figure 6, bar chart), suggesting that the key predators in bipartite networks mainly benefited from the abundant food supply. In agreement with the high resourcehigh stochasticity hypothesis of Dini-Andreote et al. (2015), our results indicate that areas of high rhizodeposition rather indiscriminately stimulated microbial growth and reproduction. This phenomenon is reflected in the high average degree and number of links between protists and prokaryotes in the lateral root network compared to networks of younger root regions.

A further microbial hotspot along the root axis are sites of lateral root emergence. In maize, the endodermis of the parent root gives rise to the epidermis of the lateral root (Lloret and Casero, 2002). Enzymes, defense compounds and other secondary metabolites are released at these sites by the breakage of lateral root primordia as earliest lateral roots through the outer root cell cortex (Ashford and McCully, 1973; 
Van Egeraat, 1975; Lloret and Casero, 2002) and were shown to attract a specific suite of microbial consumers (Jaeger et al., 1999; Baudoin et al., 2002; Park et al., 2004). Beta diversity of prokaryotes in the zone of lateral root emergence showed much less variability than in the root hair region (Figure 3 ), and their low evenness reflects a competitive community shift toward dominance of few taxa, both indicating the onset of deterministic processes of community assembly as energy supply from the root hair region had ceased. The much lower number of co-occurring taxa of the empirical network also points to a reduced availability of resources compared to the root hair region. The trophic network showed few correlations to potential prey bacteria, still indicating the absence of top-down control. While the quantity and composition of root metabolites has a direct influence on prokaryote communities, the latter in turn is expected to feed back on the community structure of their cercozoan consumers (Matz and Kjelleberg, 2005; Jousset, 2012; Song et al., 2015). The smaller ordination space in NMDS covered by prokaryotes in bulk soil and in older root sections as compared to protists likely reflects these indirect relationships (Figure 3B).The first-order laterals of maize are mostly short and reach their final length within 2-3 days (Fusseder, 1987; Pagès and Pellerin, 1994; Hochholdinger, 2009). Rhizodeposition occurs only at the distal root tips, because the fully developed root endodermis and exodermis prevent losses of cell compounds in this region (Neumann and Römheld, 2012). The scarcity of resource supply from rhizodeposition likely continues to favor deterministic processes of community assembly. Evidence comes from variation of beta diversity, which was lowest of all root regions, and the community structure showed no overlap with bulk soil communities (Figure 3A), demonstrating that a consistent root microbiome finally assembled. Co-occurrence network complexity considerably increased compared to root primordia, and the number of negative co-occurrences also rose, potentially indicating competitive exclusion. The bipartite trophic network between prokaryotes and protists on lateral roots showed clear phylogenetic patterns, as would be expected if different prokaryote taxa evolved different defensive traits that are phylogenetically clustered (Matz and Kjelleberg, 2005; Jousset, 2012; Song et al., 2015). Targeted experiments on protist-bacteria interactions demonstrate strong selection effects favoring grazing-resistant taxa (Koh et al., 2012), while reducing the dominance of fast-growing, less-defended competitors (Jousset et al., 2008).

Due to the increasingly applied role of rhizosphere microbiomes for enhancing plant productivity, a deeper mechanistic understanding of microbial assembly processes appears crucial. Community assembly in the rhizosphere is often solely viewed from the plant perspective as an increasingly plantdriven selection of microbial taxa from bulk soil to rhizosphere and rhizoplane. Our study emphasizes the importance of microbial interactions and the dynamic nature of the processes related to microbiome assembly along the root axis. Already the microbial communities on root tips differed significantly from bulk soil, but high variation in beta diversity showed substantial randomness in community structure between individual root tips. Contrary to our expectations, randomness persisted in the root hair zone, where community networks indicate rather indiscriminate growth of most taxa due to abundant resource supply. On the contrary, depletion of resources reduced variation within rhizosphere microbiomes and favored more structured network topologies, suggesting important roles of competition and predation in rhizosphere microbiome assembly.

\section{DATA AVAILABILITY STATEMENT}

The data have been submitted to the European Nucleotide Archive and are available under the accession number PRJEB40690.

\section{AUTHOR CONTRIBUTIONS}

MB designed the study. DV did the experimental framework. LR conducted the experiment, prepared the samples for sequencing, and performed the bioinformatics with the help of $\mathrm{KD}$ and data analysis assisted by KF (network analysis). BS, YC, and RS provided sequences of prokaryota. $\mathrm{MB}$ and LR wrote the manuscript with assistance of MW. PY and FH contributed significantly with their expertise on maize root development and exudation. All authors read and approved the manuscript.

\section{FUNDING}

We acknowledge the DFG (German Research Foundation) for funding the priority program 2089 Rhizosphere Spatiotemporal Organization - A Key to Rhizosphere Functions (project number 403635931).

\section{ACKNOWLEDGMENTS}

We acknowledge Caroline Marcon and Frank Hochholdinger (University of Bonn) for providing maize seeds used in this experiment.

\section{SUPPLEMENTARY MATERIAL}

The Supplementary Material for this article can be found online at: https://www.frontiersin.org/articles/10.3389/fmicb. 2021.614501/full\#supplementary-material 


\section{REFERENCES}

Anderson, T.-H., and Domsch, K. H. (1985). Maintenance carbon requirements of actively-metabolizing microbial populations under in situ conditions. Soil Biol. Biochem. 17, 197-203. doi: 10.1016/0038-0717(85) 90115-4

Andrews, J. H., and Harris, R. F. (2000). The ecology and biogeography of microorganisms on plant surfaces. Annu. Rev. Phytopathol. 38, 145-180. doi: 10.1146/annurev.phyto.38.1.145

Apprill, A., Mcnally, S., Parsons, R., and Weber, L. (2015). Minor revision to V4 region SSU rRNA 806R gene primer greatly increases detection of SAR11 bacterioplankton. Aquat. Microb. Ecol. 75, 129-137. doi: 10.3354/ame 01753

Aronesty, E. (2011). ea-utils: "Command-Line Tools for Process-ing Biological Sequencing Data. Available online at: http://expressionanalysis.github.io/eautils/ (accessed January, 2020).

Ashford, A. E., and McCully, M. E. (1973). Histochemical localization of $\beta$ glycosidases in roots ofZea mays. Protoplasma 77, 411-425. doi: 10.1007/ bf01275718

Badri, D. V., and Vivanco, J. M. (2009). Regulation and function of root exudates. Plant Cell Environ. 32, 666-681. doi: 10.1111/j.1365-3040.2009.01926.x

Baetz, U., and Martinoia, E. (2014). Root exudates: the hidden part of plant defense. Trends Plant Sci. 19, 90-98. doi: 10.1016/j.tplants.2013.11.006

Bais, H., Weir, T., Perry, L., Gilroy, S., and Vivanco, J. (2006). The role of root exudates in rhizosphere interactions with plants and other organisms. Annu. Rev. Plant Biol. 57, 233-266. doi: 10.1146/annurev.arplant.57.032905.105159

Barnard, A. M. L., Bowden, S. D., Burr, T., Coulthurst, S. J., Monson, R. E., and Salmond, G. P. C. (2007). Quorum sensing, virulence and secondary metabolite production in plant soft-rotting bacteria. Trans. R. Soc. B Biol. Sci. 362, 1165-1183. doi: 10.1098/rstb.2007.2042

Baudoin, E., Benizri, E., and Guckert, A. (2002). Impact of growth stage on the bacterial community structure along maize roots, as determined by metabolic and genetic fingerprinting. Appl. Soil Ecol. 19, 135-145. doi: 10.1016/S09291393(01)00185-8

Bauer, W., and Mathesius, U. (2004). Plant responses to bacterial quorum sensing signals. Curr. Opin. Plant Biol. 7, 429-433. doi: 10.1016/j.pbi.2004.05.008

Benizri, E., Nguyen, C., Piutti, S., Slezack-Deschaumes, S., and Philippot, L. (2007). Additions of maize root mucilage to soil changed the structure of the bacterial community. Soil Biol. Biochem. 39, 1230-1233. doi: 10.1016/j.soilbio.2006.12. 026

Berg, G., Grube, M., Schloter, M., and Smalla, K. (2014). Unraveling the plant microbiome: looking back and future perspectives. Front. Microbiol. 5:148. doi: $10.3389 /$ fmicb. 2014.00148

Blagodatskaya, E. V., Blagodatsky, S. A., Anderson, T. H., and Kuzyakov, Y. (2009). Contrasting effects of glucose, living roots and maize straw on microbial growth kinetics and substrate availability in soil. Eur. J. Soil Sci. 60, 186-197. doi: 10.1111/j.1365-2389.2008.01103.x

Blagodatskaya, E. V., and Kuzyakov, Y. (2013). Active microorganisms in soil: critical review of estimation criteria and approaches. Soil Biol. Biochem. 67, 192-211. doi: 10.1016/j.soilbio.2013.08.024

Boddy, E., Hill, P. W., Farrar, J., and Jones, D. L. (2007). Fast turnover of low molecular weight components of the dissolved organic carbon pool of temperate grassland field soils. Soil Biol. Biochem. 39, 827-835. doi: 10.1016/ j.soilbio.2006.09.030

Bolger, A. M., Lohse, M., and Usadel, B. (2014). Trimmomatic: a flexible trimmer for Illumina sequence data. Bioinformatics 30:btu170. doi: 10.1093/ bioinformatics/btu170

Bonkowski, M., and Clarholm, M. (2012). Stimulation of plant growth through interactions of bacteria and protozoa: testing the auxillary microbial loop hypothesis. Acta Protozool. 51, 237-247. doi: 10.4467/16890027AP.12.019.0765

Bouffaud, M.-L., KyselkovÁ, M., Gouesnard, B., Grundmann, G., Muller, D., and MoËNne-Loccoz, Y. (2012). Is diversification history of maize influencing selection of soil bacteria by roots? Mol. Ecol. 21, 195-206. doi: 10.1111/j.1365294X.2011.05359.x

Bulgarelli, D., Garrido-Oter, R., Munch, P. C., Weiman, A., Droge, J., Pan, Y., et al. (2015). Structure and function of the bacterial root microbiota in wild and domesticated barley. Cell Host Microbe 17, 392-403. doi: 10.1016/j.chom. 2015.01.011
Bulgarelli, D., Rott, M., Schlaeppi, K., van Themaat, E., Ahmadinejad, N., Assenza, F., et al. (2012). Revealing structure and assembly cues for Arabidopsis rootinhabiting bacterial microbiota. Nature 488, 91-95. doi: 10.1038/nature11336

Camacho, C., Coulouris, G., Avagyan, V., Ma, N., Papadopoulos, J., Bealer, K., et al. (2009). BLAST+: architecture and applications. BMC Bioinform. 10:421. doi: 10.1186/1471-2105-10-421

Caporaso, J. G., Lauber, C. L., Walters, W. A., Berg-lyons, D., Lozupone, C. A., Turnbaugh, P. J., et al. (2011). Global patterns of 16S rRNA diversity at a depth of millions of sequences per sample. Proc. Natl. Acad. Sci. U.S.A. 108, 4516-4522. doi: 10.1073/pnas.1000080107

Chaparro, J. M., Badri, D. V., and Vivanco, J. M. (2014). Rhizosphere microbiome assemblage is affected by plant development. ISME J. 8, 790-803. doi: 10.1038/ ismej.2013.196

Corno, G., and Jürgens, K. (2008). Structural and functional patterns of bacterial communities in response to protist predation along an experimental productivity gradient. Environ. Microbiol. 10, 2857-2871. doi: 10.1111/j.14622920.2008.01713.x

de Moraes, M. T., Debiasi, H., Franchini, J. C., Bonetti, J. D. A., Levien, R., Schnepf, A., et al. (2019). Mechanical and hydric stress effects on maize root system development at different soil compaction levels. Front. Plant Sci. 10:1358. doi: 10.3389/fpls.2019.01358

Deng, Y., Jiang, Y., Yang, Y., He, Z., Luo, F., and Zhou, J. (2012). Molecular ecological network analyses. BMC Bioinform. 13:113. doi: 10.1186/1471-210513- 113

Denison, R., Bledsoe, C., Kahn, M., O’Gara, F., Simms, E., and Thomashow, L. (2003). Cooperation in the rhizosphere and the "free-rider" problem. Ecology 84, 838-845. doi: 10.1890/0012-9658(2003)084[0838:citrat]2.0.co;2

Di Battista-Leboeuf, C., Benizri, E., Corbel, G., Piutti, S., and Guckert, A. (2003). Distribution of Pseudomonas sp. populations in relation to maize root location and growth stage. Agronomie 23, 441-446. doi: 10.1051/agro:2003017

Dini-Andreote, F., Stegen, J. C., van Elsas, J. D., and Salles, J. F. (2015). Disentangling mechanisms that mediate the balance between stochastic and deterministic processes in microbial succession. Proc. Natl. Acad. Sci. U.S.A. 112, E1326-E1332. doi: 10.1073/pnas.1414261112

Dupuy, L. X., and Silk, W. K. (2016). Mechanisms of early microbial establishment on growing root surfaces. Vadose Zone J. 15, 1-13. doi: 10.2136/vzj2015.06.0094

Edgar, R. C., Haas, B. J., Clemente, J. C., Quince, C., and Knight, R. (2011). UCHIME improves sensitivity and speed of chimera detection. Bioinformatics 27, 2194-2200. doi: 10.1093/bioinformatics/btr381

Edwards, J., Johnson, C., Santos-Medellin, C., Lurie, E., Podishetty, N. K., Bhatnagar, S., et al. (2015). Structure, variation, and assembly of the rootassociated microbiomes of rice. Proc. Natl. Acad. Sci. U.S.A. 112, E911-E920. doi: 10.1073/pnas.1414592112

Fan, K., Cardona, C., Li, Y., Shi, Y., Xiang, X., Shen, C., et al. (2017). Rhizosphere-associated bacterial network structure and spatial distribution differ significantly from bulk soil in wheat crop fields. Soil Biol. Biochem. 113, 275-284. doi: 10.1016/j.soilbio.2017.06.020

Farrar, J., Hawes, M., Jones, D., and Lindow, S. (2003). How roots control the flux of carbon to the rhizosphere. Ecology 84, 827-837. doi: 10.1890/0012-9658(2003) 084[0827:hrctfo]2.0.co;2

Fierer, N., Bradford, M. A., and Jackson, R. B. (2007). Toward an ecological classification of soil bacteria. Ecology 88, 1354-1364. doi: 10.1890/05-1839

Fiore-Donno, A. M., Richter-Heitmann, T., and Bonkowski, M. (2020). Contrasting responses of protistan plant parasites and phagotrophs to ecosystems, land management and soil properties. Front. Microbiol. 11:1823. doi: $10.3389 /$ fmicb. 2020.01823

Fiore-Donno, A. M., Rixen, C., Rippin, M., Glaser, K., Samolov, E., Karsten, U., et al. (2018). New barcoded primers for efficient retrieval of cercozoan sequences in high-throughput environmental diversity surveys, with emphasis on worldwide biological soil crusts. Mol. Ecol. Res. 18, 229-239. doi: 10.1111/1755-0998.12729

Flues, S., Bass, D., and Bonkowski, M. (2017). Grazing of leaf-associated Cercomonads (Protists: Rhizaria: Cercozoa) structures bacterial community composition and function. Environ. Microbiol. 19, 3297-3309. doi: 10.1111/ 1462-2920.13824

Friesen, M. L., Porter, S. S., Stark, S. C., von Wettberg, E. J., Sachs, J. L., and Martinez-Romero, E. (2011). Microbially mediated plant functional traits. Annu. Rev. Ecol. Evol. Syst. 42, 23-46. doi: 10.1146/annurev-ecolsys-102710145039 
Fukami, T. (2015). Historical contingency in community assembly: integrating niches, species pools, and priority effects. Annu. Rev. Ecol. Evol. Syst. 46, 1-23. doi: 10.1146/annurev-ecolsys-110411-160340

Fusseder, A. (1987). The longevity and activity of the primary root of maize. Plant Soil 101, 257-265. doi: 10.1007/BF02370653

Guillou, L., Bachar, D., Audic, S., Bass, D., Berney, C., Bittner, L., et al. (2013). The protist ribosomal reference database (PR2): a catalog of unicellular eukaryote small sub-unit rRNA sequences with curated taxonomy. Nucleic Acids Res. 41, D597-D604. 10.1093/nar/gks1160

Hartmann, A., and Schikora, A. (2012). Quorum sensing of bacteria and transkingdom interactions of $\mathrm{N}$-acyl homoserine lactones with eukaryotes. J. Chem. Ecol. 38, 704-713. doi: 10.1007/s10886-012-0141-7

Hartmann, A., Schmid, M., van Tuinen, D., and Berg, G. (2009). Plant-driven selection of microbes. Plant Soil 321, 235-257. doi: 10.1007/s11104-008-9814-y

Hawes, M. (1991). "Living plant cells released from the root cap: A regulator of microbial populations in the rhizosphere?", in The Rhizosphere and Plant Growth, eds D. L. Keister and P. B. Cregan (Dordrecht: Kluwer Academic Publishers), 51-59. doi: 10.1007/978-94-011-3 336-4_6

Hawes, M. C., Curlango-Rivera, G., Xiong, Z., and Kessler, J. O. (2012). Roles of root border cells in plant defense and regulation of rhizosphere microbial populations by extracellular DNA 'trapping'. Plant Soil 355, 1-16. doi: 10.1007/ s11104-012-1218-3

Henkes, G. J., Kandeler, E., Marhan, S., Scheu, S., and Bonkowski, M. (2018). Interactions of mycorrhiza and protists in the rhizosphere systemically alter microbial community composition, plant shoot-to-root ratio and within-root system nitrogen allocation. Front. Environ. Sci. 6:117. doi: 10.3389/fenvs.2018. 00117

Hirsch, P. R., and Mauchline, T. H. (2012). Who's who in the plant root microbiome? Nat. Biotech. 30, 961-962. doi: 10.1038/nbt.2387

Hochholdinger, F. (2009). "The maize root system: morphology, anatomy, and genetics," in Handbook of Maize: Its Biology, eds J. L. Bennetzen and S. C. Hake (New York, NY: Springer).

Humphris, S. N., Bengough, A. G., Griffiths, B. S., Kilham, K., Rodger, S., Stubbs, V., et al. (2005). Root cap influences root colonisation by Pseudomonas fluorescens SBW25 on maize. FEMS Microbiol. Ecol. 54, 123-130. doi: 10.1016/j.femsec. 2005.03.005

Iijima, M., Griffiths, B., and Bengough, A. (2000). Sloughing of cap cells and carbon exudation from maize seedling roots in compacted sand. New Phytol. 145, 477-482. doi: 10.1046/j.1469-8137.2000.00595.x

Jaeger, C. I., Lindow, S., Miller, W., Clark, E., and Firestone, M. (1999). Mapping of sugar and amino acid availability in soil around roots with bacterial sensors of sucrose and tryptophan. Appl. Environ. Microbiol. 65, 2685-2690. doi: 10.1128/ aem.65.6.2685-2690.1999

Jousset, A. (2012). Ecological and evolutive implications of bacterial defences against predators. Environ. Microbiol. 14, 1830-1843. doi: 10.1111/j.1462-2920. 2011.02627.x

Jousset, A., and Bonkowski, M. (2010). The model predator Acanthamoeba castellanii induces the production of 2,4 , DAPG by the biocontrol strain Pseudomonas fluorescens Q2-87. Soil Biol. Biochem. 42, 1647-1649. doi: 10. 1016/j.soilbio.2010.05.018

Jousset, A., Lara, E., Wall, L. G., and Valverde, C. (2006). Secondary metabolites help biocontrol strain Pseudomonas fluorescens CHA0 to escape protozoan grazing. Appl. Environ. Microbiol. 72, 7083-7090. doi: 10.1128/aem.00557-06

Jousset, A., Rochat, L., Pechy-Tarr, M., Keel, C., Scheu, S., and Bonkowski, M. (2009). Predators promote defence of rhizosphere bacterial populations by selective feeding on non-toxic cheaters. ISME J. 3, 666-674. doi: 10.1038/ismej. 2009.26

Jousset, A., Rochat, L., Scheu, S., Bonkowski, M., and Keel, C. (2010). Predator-prey chemical warfare determines the expression of biocontrol genes by rhizosphereassociated Pseudomonas fluorescens. Appl. Environ. Microbiol. 76, 5263-5268. doi: 10.1128/aem.02941-09

Jousset, A., Scheu, S., and Bonkowski, M. (2008). Secondary metabolite production facilitates establishment of rhizobacteria by reducing both protozoan predation and the competitive effects of indigenous bacteria. Funct. Ecol. 22, 714-719. doi: 10.1111/j.1365-2435.2008.01411.x

Knee, E. M., Gong, F.-C., Gao, M., Teplitski, M., Jones, A. R., Foxworthy, A., et al. (2001). Root mucilage from pea and its utilization by rhizosphere bacteria as a sole carbon source. Mol. Plant Microbe Interact. 14, 775-784. doi: 10.1094/ mpmi.2001.14.6.775

Koh, K. S., Matz, C., Tan, C. H., Le, H. L., Rice, S. A., Marshall, D. J., et al. (2012). Minimal increase in genetic diversity enhances predation resistance. Mol. Ecol. 21, 1741-1753. doi: 10.1111/j.1365-294X.2011.05415.x

Li, B., Li, Y.-Y., Wu, H.-M., Zhang, F.-F., Li, C.-J., Li, X.-X., et al. (2016). Root exudates drive interspecific facilitation by enhancing nodulation and $\mathrm{N}_{2}$ fixation. Proc. Natl. Acad. Sci. 113, 6496-6501. doi: 10.1073/pnas.1523 580113

Lloret, P. G., and Casero, P. J. (2002). "Lateral Root Initiation,” in Plant Roots-the Hidden Half, eds Y. Waisel, A. Eshel, and U. Kafkafi (New York, NY: Marcel Dekker), 127-155. doi: 10.1201/9780203909423.ch8

Lugtenberg, B. J., Dekkers, L., and Bloemberg, G. V. (2001). Molecular determinants of rhizosphere colonization by Pseudomonas. Annu. Rev. Phytopathol. 39, 461-490. doi: 10.1146/annurev.phyto.39.1.461

Lundberg, D. S., Lebeis, S. L., Paredes, S. H., Yourstone, S., Gehring, J., Malfatti, S., et al. (2012). Defining the core Arabidopsis thaliana root microbiome. Nature 488, 86-90. doi: 10.1038/nature 11237

Maloney, P. E., van Bruggen, A. H. C., and Hu, S. (1997). Bacterial community structure in relation to the carbon environments in lettuce and tomato rhizospheres and in bulk soil. Microb. Ecol. 34, 109-117. doi: 10.1007/ s002489900040

Martin, M. (2011). Cutadapt removes adapter sequences from high-throughput sequencing reads. EMBnet. J. 17, 10-12. doi: 10.14806/ej.17.1.200

Mathesius, U., Mulders, S., Gao, M., Teplitski, M., Caetano-Anolles, G., Rolfe, B., et al. (2003). Extensive and specific responses of a eukaryote to bacterial quorum-sensing signals. Proc. Natl. Acad. Sci. U.S.A. 100, 1444-1449. doi: 10. 1073/pnas.262672599

Matz, C., and Jurgens, K. (2003). Interaction of nutrient limitation and protozoan grazing determines the phenotypic structure of a bacterial community. Microb. Ecol. 45, 384-398. doi: 10.1007/s00248-003-2000-0

Matz, C., and Kjelleberg, S. (2005). Off the hook-how bacteria survive protozoan grazing. Trends Microbiol. 13, 302-307. doi: 10.1016/j.tim.2005.05.009

Naher, U. A., Radziah, O., Halimi, M. S., Shamsuddin, Z. H., and Razi, I. M. (2008). Effect of inoculation on root exudates carbon sugar and amino acids production of different rice varieties. Res. J. Microbiol. 3, 580-587. doi: 10.3923/jm.2008. 580.587

Nemergut, D. R., Schmidt, S. K., Fukami, T., O’Neill, S. P., Bilinski, T. M., Stanish, L. F., et al. (2013). Patterns and processes of microbial community assembly. Microbiol. Mol. Biol. Rev. 77, 342-356. doi: 10.1128/mmbr.00051-12

Neumann, G., and Römheld, V. (2012). "Rhizosphere chemistry in relation to plant nutrition," in Marschner's Mineral Nutrition of Higher Plants, 3rd Edn, ed. P. Marchner (Amsterdam: Academic Press).

Nguyen, C. (2003). Rhizodeposition of organic C by plants: mechanisms and controls. Agronomie 23, 375-396. doi: 10.1051/agro:2003011

Oger, P. M., Mansouri, H., Nesme, X., and Dessaux, Y. (2004). Engineering root exudation of lotus toward the production of two novel carbon compounds leads to the selection of distinct microbial populations in the rhizosphere. Microb. Ecol. 47, 96-103. doi: 10.1007/s00248-003-2012-9

Osborn, H. M. I., Lochey, F., Mosley, L., and Read, D. (1999). Analysis of polysaccharides and monosaccharides in the root mucilage of maize (Zea mays L.) by gas chromatography. J. Chromatogr. A 831, 267-276. doi: 10.1016/S00219673(98)00935-2

Oyserman, B. O., Medema, M. H., and Raaijmakers, J. M. (2018). Road MAPs to engineer host microbiomes. Curr. Opin. Microbiol. 43, 46-54. doi: 10.1016/j. mib.2017.11.023

Pagès, L., and Pellerin, S. (1994). Evaluation of parameters describing the root system architecture of field grown maize plants (Zea mays L.). Plant Soil 164, 169-176. doi: 10.1007/BF00010068

Park, W. J., Hochholdinger, F., and Gierl, A. (2004). Release of the benzoxazinoids defense molecules during lateral- and crown root emergence in Zea mays. J. Plant Physiol. 161, 981-985. doi: 10.1016/j.jplph.2004.01.005

Peiffer, J. A., Spor, A., Koren, O., Jin, Z., Tringe, S. G., Dangl, J. L., et al. (2013). Diversity and heritability of the maize rhizosphere microbiome under field conditions. Proc. Natl. Acad. Sci. 110, 6548-6553. doi: 10.1073/pnas. 1302837110

Perez-Jaramillo, J. E., Carrion, V. J., Bosse, M., Ferrao, L. F. V., de Hollander, M., Garcia, A. A. F., et al. (2017). Linking rhizosphere microbiome composition 
of wild and domesticated Phaseolus vulgaris to genotypic and root phenotypic traits. ISME J. 11, 2244-2257. doi: 10.1038/ismej.2017.85

Perez-Jaramillo, J. E., Mendes, R., and Raaijmakers, J. M. (2016). Impact of plant domestication on rhizosphere microbiome assembly and functions. Plant Mol. Biol. 90, 635-644. doi: 10.1007/s11103-015-0337-7

Piñeros, M. A., Magalhaes, J. V., Carvalho Alves, V. M., and Kochian, L. V. (2002). The physiology and biophysics of an aluminum tolerance mechanism based on root citrate exudation in maize. Plant Physiol. 129, 1194-1206. doi: 10.1104/pp. 002295

R Core Team (2018). "R: a language and environment for statistical computing," in $R$ Foundation for Statistical Computing (Vienna).

Riley, M. A., and Wertz, J. E. (2002). Bacteriocins: evolution, ecology, and application. Annu. Rev. Microbiol. 56, 117-137. doi: 10.1146/annurev.micro.56. 012302.161024

Rognes, T., Flouri, T., Nichols, B., Quince, C., and Mahé, F. (2016). VSEARCH: a versatile open source tool for metagenomics. PeerJ 4 4:e2584. doi: 10.7717/peerj. 2584

Rosenberg, K., Bertaux, J., Krome, K., Hartmann, A., Scheu, S., and Bonkowski, M. (2009). Soil amoebae rapidly change bacterial community composition in the rhizosphere of Arabidopsis thaliana. ISME J. 3, 675-684. doi: 10.1038/ismej. 2009.11

Rossmann, M., Pérez-Jaramillo, J. E., Kavamura, V. N., Chiaramonte, J. B., Dumack, K., Fiore-Donno, A. M., et al. (2020). Multitrophic interactions in the rhizosphere microbiome of wheat: from bacteria and fungi to protists. FEMS Microbiol. Ecol. 96, fiaa032. doi: 10.1093/femsec/fiaa032

Schloss, P. D., Westcott, S. L., Ryabin, T., Hall, J. R., Hartmann, M., Hollister, E. B., et al. (2009). Introducing mothur : open-source, platform-independent, community-supported software for describing and comparing microbial communities. Appl. Environ. Microbiol. 75, 7537-7541. doi: 10.1128/AEM. 01541-09

Schönwitz, R., and Ziegler, H. (1989). Interaction of maize roots and rhizosphere microorganisms. Zeitschrift für Pflanzenernährung und Bodenkunde 152, 217222. doi: 10.1002/jpln.19891520213

Shannon, P., Markiel, A., Ozier, O., Baliga, N. S., Wang, J. T., Ramage, D., et al. (2003). Cytoscape: a software environment for integrated models of biomolecular interaction networks. Genome Res. 13, 2498-2504. doi: 10.1101/ gr. 1239303

Shaw, L. J., Morris, P., and Hooker, J. E. (2006). Perception and modification of plant flavonoid signals by rhizosphere microorganisms. Environ. Microbiol. 8, 1867-1880. doi: 10.1111/j.1462-2920.2006.01141.x

Shi, S., Nuccio, E., Herman, D. J., Rijkers, R., Estera, K., Li, J., et al. (2015). Successional trajectories of rhizosphere bacterial communities over consecutive seasons. mBio 6:e00746. doi: 10.1128/mBio.00746-15

Song, C., Mazzola, M., Cheng, X., Oetjen, J., Alexandrov, T., Dorrestein, P., et al. (2015). Molecular and chemical dialogues in bacteria-protozoa interactions. Sci. Rep. 5:12837. doi: $10.1038 /$ srep 12837
Sprockett, D., Fukami, T., and Relman, D. A. (2018). Role of priority effects in the early-life assembly of the gut microbiota. Nat. Rev. Gastroenterol. Hepatol. 15, 197-205. doi: 10.1038/nrgastro.2017.173

Steinkellner, S., Lendzemo, V., Langer, I., Schweiger, P., Khaosaad, T., Toussaint, J.-P., et al. (2007). Flavonoids and strigolactones in root exudates as signals in symbiotic and pathogenic plant-fungus interactions. Mol. 12, 1290-1306. doi: 10.3390/12071290

Tovi, N., Frenk, S., Hadar, Y., and Minz, D. (2019). Host specificity and spatial distribution preference of three Pseudomonas isolates. Front. Microb. 9:3263. doi: 10.3389/fmicb.2018.03263

van Dam, N. M., and Bouwmeester, H. J. (2016). Metabolomics in the rhizosphere: tapping into belowground chemical communication. Trends Plant Sci. 21, 256-265. doi: 10.1016/j.tplants.2016. 01.008

van der Heijden, M. G., and Schlaeppi, K. (2015). Root surface as a frontier for plant microbiome research. Proc. Natl. Acad. Sci. U.S.A. 112, 2299-2300. doi: 10.1073/pnas.1500709112

Van Egeraat, A. W. S. M. (1975). Exudation of ninhydrin-positive compounds by pea-seedling roots: a study of the sites of exudation and of the composition of the exudate. Plant Soil 42, 37-47. doi: 10.1007/BF02 186972

Walker, T., Bais, H., Grotewold, E., and Vivanco, J. (2003). Root exudation and rhizosphere biology. Plant Physiol. 132, 44-51. doi: 10.1104/pp.102.019661

Watt, M., Silk, W., and Passioura, J. (2006). Rates of root and organism growth, soil conditions, and temporal and spatial development of the rhizosphere. Ann. Bot. 97, 839-855. doi: 10.1093/aob/mcl028

Zelenev, V., Van Bruggen, A., and Semenov, A. (2005). Modeling wave-like dynamics of oligotrophic and copiotrophic bacteria along wheat roots in response to nutrient input from a growing root tip. Ecol. Model. 188, 404-417. doi: 10.1016/j.ecolmodel.2005.01.046

Zhou, J., Deng, Y., Luo, F., He, Z., and Yang, Y. (2011). Phylogenetic molecular ecological network of soil microbial communities in response to elevated CO2. mBio 2:e0122-11. doi: 10.1128/mBio.00122-11

Conflict of Interest: The authors declare that the research was conducted in the absence of any commercial or financial relationships that could be construed as a potential conflict of interest.

Copyright (c) 2021 Rüger, Feng, Dumack, Freudenthal, Chen, Sun, Wilson, Yu, Sun, Deng, Hochholdinger, Vetterlein and Bonkowski. This is an open-access article distributed under the terms of the Creative Commons Attribution License (CC BY). The use, distribution or reproduction in other forums is permitted, provided the original author(s) and the copyright owner(s) are credited and that the original publication in this journal is cited, in accordance with accepted academic practice. No use, distribution or reproduction is permitted which does not comply with these terms. 